\title{
Characterisation of bioactive compounds in berry juices by traditional photometric and modern microplate methods
}

\author{
Anna Horszwald and Wilfried Andlauer* \\ Institute of Life Technologies, University of Applied Sciences Valais, Sion, Switzerland
}

Received 11 February 2011; accepted 13 April 2011

\begin{abstract}
Berries and berry production are an important economic factor. Berries contain nutritive components but also pertinent non-nutritive bioactives like phenolic-type phytochemicals. It is well accepted that consumption of bioactives from berries prevent or delay chronic and degenerative diseases. Therefore, a thorough evaluation of bioactive compounds is of utmost interest to assess berry quality. The most accepted photometric methods for the determination of bioactive compounds are presented for conventional cuvette and modern microplate applications. All methods are precisely described and advantages of the microplate methods are discussed. Cuvette and microplate methods show slightly different sensitivity and limits of detection depending on concentrations used for reagents and standard compounds. In general, with microplate methods reagents can be saved up to 12-fold and less time to perform the analyses is needed (up to factor 33). Applicability of all the methods has been shown with selected berry juices. Among all analysed commercial juices, blueberry juice had the highest content of total polyphenols, flavonoids, proanthocyanidins, while the lowest content was observed in pomegranate juice. Blueberry juice had also the highest antioxidant capacity measured by TEAC by ABTS, FRAP and ORAC method when compared to the cranberry, açai, goji and pomegranate juice.
\end{abstract}

Keywords: Blueberry, cranberry, goji, açai, pomegranate, bioactive compounds, photometric methods, antioxidant activity, microplate

\section{Introduction}

Berries comprise the major proportion of the fruits present in European diet [1,2]. The worldwide production of berries in 2009 was more than 5.3 million $t$ (mainly blueberry, cranberry, raspberry and strawberry) with a harvest area of 440148 ha [3]. Production of these fruits among European Union countries consisted of approximately $33 \%$ of the global production and $35 \%$ of the global harvested area. In general, berries can be consumed freshly or as processed fruits mainly as juices, jams, jellies, wine as well as additives to foodstuffs [4]. Their presence in the diet makes an important contribution to the intake of health promoting compounds [1]. It was confirmed that besides of nutritive dietary components including vitamins, minerals, organic acids, dietary fibres, sugars and unsaturated fats [5], berries are an excellent source of phenolic-type phytochemicals like flavonoids (anthocyanins, flavonols, flavanols), tannins (proanthocyanidins, ellagitannins, gallotannins), phenolic acids (hydroxybenzoic and hydroxycinnamic acid derivates), stilbenoids (resveratrol) and lignans [6]. Consumption of bioactives present in berries may lead to the prevention or delay of many chronic and degenerative diseases including cancers, cardiovascular disease, immune system decline and certain neurological disorder [1,7-11]. Taking this into consideration, it can be stated that thorough evaluation of certain compounds in commercially available products might influence the consumers' choice of products [12]. Nowadays, antioxidant capacity of products gained extreme popularity due to

*Corresponding author: Wilfried Andlauer, Institute of Life Technologies, University of Applied Sciences Valais, Route du Rawyl 47, CH-1950 Sion, Switzerland. Tel.: +41 (0) 2760686 37; Fax: +41 (0) 2760686 15; E-mail: wilfried.andlauer@ hevs.ch. 
the progressive development of analytical assays and consumers are very sensible concerning these information. However, quantification of bioactive compounds as well as determination of antioxidant capacity of berry samples is elusive due to the complexity of their composition and the diversity of antioxidants [13]. There are also numerous methodological aspects influencing the final results [14], among them the concentration of oxidation source, ratio antioxidant/radicals, interaction between target compound and formed complexes, as well as the environment of the reaction. Additionally, aspects connected with processing of berries that influence the content of bioactives should be also taken into consideration.

Nevertheless, a concern of economical aspects like time-consuming preparation and expensive processes should be made. For laboratories, reduction of analysis costs is one of the most important goals. Among a broad range of methods used for the determination of phenolic-type phytochemicals, especially spectrophotometric and spectrofluorimetric ones seem to be simple and effortless to perform. Nowadays, microplate measurements are widely accepted and used in research and routine laboratories. Microplate methods enable more automated working with less reagent and sample quantities. Consequently, microplate methods are quicker and less cost intensive.

Another possibility for an automation and standardization of determination methods for antioxidant capacity regarding TEAC by ABTS and DPPH [15] and reducing capacity with Folin-Ciocalteu reagent [16] has been presented. This research group applied a flow injection analysis system, known as a simple and cheap method for photometric analysis. Recently, comparable to flow injection system, RESAC method has been proposed and validated for a rapid measurement of antioxidant capacity from plant matrices [17]. However, specific equipment appropriate for determination of antioxidant capacity is needed for these methods. Moreover, well accepted methods like ORAC cannot be employed by flow injection systems.

The main goal of this publication is to present some of the most accepted methods for the determination of bioactive compounds and to adapt them to modern microplate measurement. Methods for the determination of total polyphenols, total flavonoids, and total proanthocyanidins content as well as antioxidant capacity (TEAC with application of ABTS radical cation and DPPH radical, FRAP and ORAC) in plant matrices are selected.

Sensitivity, limit of detection, costs and time needed per analysis for the classical cuvette and the modern microplates methods have been compared. On the basis of selected berry juices, the applicability of cuvette tests and the adapted microplate methods has been presented. All methods are precisely described and advantages of the microplate methods are discussed.

\section{Material and methods}

\subsection{Reagents}

All reagents used were of analytical grade. Folin-Ciocalteu's phenol reagent, 2,2'-azobis(2-methylpropionamidine) dihydrochloride (AAPH), (+)-catechine, sodium acetate and quercetin were obtained from Sigma-Aldrich (Switzerland). Aluminium chloride hexahydrate, 2,2-diphenyl-1-picrylhydrazyl (DPPH), gallic acid, fluorescein sodium, potassium acetate, potassium persulfate, dipotassium hydrogen phosphate dihydrate, sodium dihydrogen phosphate dehydrate, vanillin and 2,4,6-tri(2-pirydyl)-s-tiazine (TPTZ) were provided by Fluka (Germany). Iron (III) chloride hexahydrate was from Riedel-de Haën (Germany). Acetic and hydrochloric acids were purchased from Pancreac (Pancreac Quimica SA, Spain). Trolox was supplied by Acros Organics (Thermo Fisher Scientific, Geel, Belgium). Sodium carbonate was obtained from Carlo Erba Reagents SA (Val de Reuil, France). All other solvents and chemicals were obtained from Merck (Darmstadt, Germany).

\subsection{Materials}

Four different berry juices as well as a pomegranate juice were produced commercially and were purchased at a local shop (Sion, Switzerland). Whole fruit cranberry juice, whole fruit blueberry and pomegranate were supplied by Donath (Germany). Goji and açai juices were obtained from Dynamic Health Laboratorie (USA).

Portions of selected juices were centrifuged at $3.2 \times \mathrm{g}$ for $10 \mathrm{~min}$ at ambient temperature (Eppendorf Centrifuge 5810, Hamburg, Germany). Subsequently, supernatants were filtered through $0.45 \mu \mathrm{m}$ PTFE filter (Chromafil 
CA-45/25 S, Duren, Germany). Clear extracts were subjected to the analysis of bioactive compounds and antioxidant capacity by traditional cuvettes and adapted microplates methods.

\subsection{Methods}

Absorption measurements carried out in plastic cuvettes with a light path of $1 \mathrm{~cm}(2.5 \mathrm{~mL}$ and $1.5 \mathrm{~mL}$, Plastibrand, Brand, Germany) were conducted by Biochrom Libra S12 (Cambridge, England), whereas the fluorescence reading performed in a glass cuvettes were done by Carry Eclipse (Varian, Australia).

All measurements performed in microplates were recorded on Infinite M200 Pro Multimode Microplate Reader (Tecan $\mathrm{GmbH}$, Austria) equipped with two channel injector systems. These injector systems enable a quick and reproducible addition of two different reagents without any hardware changes and interruptions. Transparent microplates for absorbance readings (96-wells) and black microplates for fluorescence recordings (96-wells) were supplied by NUNC ${ }^{\mathrm{TM}}$ (Nunc A/S, Roskilde, Denmark).

\subsection{Total phenolic compounds (TPC)}

The content of total polyphenolic compounds (TPC) was determined by Folin-Ciocalteu colorimetric method and was performed according to Siriwoharn et al. [18]. For analysis in cuvettes a volume of $0.5 \mathrm{~mL}$ of appropriately diluted samples, blanks or standards was placed into test tube followed by addition of $0.5 \mathrm{~mL}$ of Folin-Ciocalteu Phenol Reagent and $7.5 \mathrm{~mL}$ of deionised water. After $10 \mathrm{~min}, 0.5 \mathrm{~mL}$ of sodium carbonate $(20 \%, \mathrm{w} / \mathrm{v})$ was added. Samples were vortexed and incubated for $20 \mathrm{~min}$ at ambient temperature in the dark for $20 \mathrm{~min}$.

TPC method was performed also in microplates and the method was modified as follows: aliquots of $15 \mu \mathrm{l}$ of appropriately diluted samples were placed into wells of microplates. Subsequently, $240 \mu l$ of Folin-Ciocalteu Phenol reagent (previously diluted with water $1: 15, \mathrm{v} / \mathrm{v}$ ) was added by injector and the mixture was incubated in dark for $10 \mathrm{~min}$ at ambient temperature. Then, $15 \mu \mathrm{l}$ of $20 \%$ sodium carbonate have been added to each well and microplate was automatically shaken before readings. The absorbance was measured at $\lambda=755 \mathrm{~nm}$.

Results were expressed as $\mathrm{g}$ of gallic acid equivalent (GAE) per $\mathrm{L}$ of juice. A blank (water) and calibration solutions using gallic acid (stock solution $1 \mathrm{mg} / \mathrm{mL}$ ) as a standard were carried out in each run. Each analysis of phenolic compounds in the extracts was done in triplicate.

\subsection{Total flavonoids content (TF)}

The total flavonoids content was determined according to a procedure proposed by Chang et al. [19]. Determination of total flavonoids was conducted in cuvettes by addition of $0.25 \mathrm{~mL}$ of appropriately diluted sample, blank or standard to $0.75 \mathrm{~mL} 95 \%$ ethanol (v/v). Subsequently, $0.05 \mathrm{~mL}$ of $10 \% \mathrm{AlCl}_{3} * 6 \mathrm{H}_{2} \mathrm{O}(\mathrm{w} / \mathrm{v})$ and $0.05 \mathrm{~mL}$ of $1 \mathrm{~mol} / \mathrm{L}$ potassium acetate were placed and mixed. At the end, $1.4 \mathrm{~mL}$ of deionised water was added.

This method was slightly modified for application of microplates. Aliquots of $25 \mu \mathrm{l}$ of respective samples were mixed with $75 \mu \mathrm{l}$ of $95 \%$ ethanol (v/v). Subsequently, $5 \mu \mathrm{l}$ of $10 \% \mathrm{AlCl}_{3} * 6 \mathrm{H}_{2} \mathrm{O}$ as well as $5 \mu \mathrm{l}$ of $1 \mathrm{~mol} / \mathrm{L}$ potassium acetate were added by the microplate reader's injector system followed by an addition of $140 \mu$ l of deionised water. The samples were vortexed and left for $30 \mathrm{~min}$ at ambient temperature. Subsequently, the absorbance of clear supernatants was recorded at $\lambda=415 \mathrm{~nm}$ against deionised water. Quercetin within the concentration from 0.5 to $0.015 \mathrm{mg} / \mathrm{mL}$ dissolved in methanol/water (4/1, v/v) was used as reference compound.

All measurements were performed in triplicate. The results obtained for analysed juices were expressed as $g$ quercetin $(\mathrm{Q})$ per $\mathrm{L}$ of juice.

\subsection{Total proanthocyanidins $(T P)$}

The content of total proanthocyanidins was performed according to Sun et al. [20]. For analysis in cuvettes, $0.25 \mathrm{~mL}$ of respective sample was mixed with $1.5 \mathrm{~mL}$ of $4 \%$ vanillin-methanol solution (w/v). Next, $0.75 \mathrm{~mL}$ of $32 \%$ hydrochloric acid were added. 
Simultaneously, the reaction was performed in microplates. To this end, the following reagents were added: $25 \mu 1$ of respective samples were mixed with $150 \mu$ l of $4 \%$ vanillin-methanol solution and $75 \mu$ l of $32 \%$ hydrochloric acid. Subsequently, the mixture was left for $15 \mathrm{~min}$ at ambient temperature. The absorbance versus prepared reagent blank containing water instead of sample was read at $\lambda=500 \mathrm{~nm}$.

Calculations were based on calibration curve obtained with (+)-catechin (CAT). Results were expressed as $g$ catechin per $\mathrm{L}$ of juice. All measurements were performed in triplicate.

\subsection{TEAC (Trolox Equivalent Antioxidant Capacity) by ABTS}

The Trolox Equivalent Antioxidant Capacity (TEAC) assay was performed as described by Re et al. [21] for cuvettes and by Silva et al. [22] for microplates with some modifications. The $7 \mathrm{mmol} / \mathrm{L}$ aqueous solution of ABTS $(10 \mathrm{~mL})$ and $51.4 \mathrm{mmolL}^{-1}$ aqueous solution of $\mathrm{K}_{2} \mathrm{~S}_{2} \mathrm{O}_{4}(0.5 \mathrm{~mL})$ were mixed in order to obtain a radical cation $\left(\mathrm{ABTS}^{\bullet+}\right.$ ) solution with absorbance value $0.7 \pm 0.05$ at $\lambda=734 \mathrm{~nm}$. For the measurements performed in cuvettes, $20 \mu \mathrm{l}$ of analysed samples was added to $1480 \mu \mathrm{l}$ of $\mathrm{ABTS}^{\bullet+}$ solution.

For analysis in microplates, aliquots of $10 \mu \mathrm{l}$ appropriate diluted samples, blanks or standard were places into microplate wells. Subsequently, the reaction and time measurement have been started with addition of $290 \mu 1$ of $\mathrm{ABTS}^{\bullet}+$ solution by the microplate reader's injector.

For microplates as well as for cuvettes, the reaction was performed at $30^{\circ} \mathrm{C}$ in dark during 6 min. After this time, the values of absorbance were recorded. The Trolox solution (stock solution $1.00 \mathrm{mmol} / \mathrm{L}$ ) was used for calibration. Obtained results were expressed as mmol Trolox Equivalents (TE) per L of juice.

\subsection{TEAC (Trolox Equivalent Antioxidant Capacity) by DPPH}

The DPPH scavenging radical assay was performed according to Brand-Williams et al. [23]. Briefly, $10 \mathrm{mg}$ of DPPH was dissolved in $250 \mathrm{~mL}$ of $80 \%$ methanol in order to obtain the DPPH solution absorbing in the range from 0.95 to 1.10 at $\lambda=517 \mathrm{~nm}$. The solution was freshly prepared before analysis.

For application of cuvettes, $200 \mu \mathrm{l}$ of appropriate diluted samples, blanks or standard were places into tube. To start the reaction, the $3 \mathrm{~mL}$ of $\mathrm{DPPH}^{\bullet}$ solution were added.

In case of microplates, $20 \mu \mathrm{l}$ of appropriate diluted samples, blanks or standard were placed into microplate wells and then $300 \mu \mathrm{l}$ of $\mathrm{DPPH}^{\bullet}$ solution were added automatically by injector.

The reaction was performed at ambient temperature during $30 \mathrm{~min}$ in the dark. Trolox within the range of 0.05 to $1.00 \mathrm{mmolL}^{-1}$ was used as a standard to calibrate the final results. The results obtained for juices were expressed and mmol Trolox Equivalents (TE) per L of juice.

\subsection{Ferric Reducing Antioxidant Potential (FRAP)}

Ferric Reducing Antioxidant Potential was conducted according to Benzie and Strain [24]. Firstly, FRAP reagent was prepared freshly before analysis. Then, $5 \mathrm{~mL}$ of $10 \mathrm{mmolL}^{-1}$ 2,4,6-tri(2-pirydyl)-s-triazine (TPTZ) in $40 \mathrm{mmolL}^{-1}$ hydrochloric acid were mixed with $5 \mathrm{~mL}$ of $20 \mathrm{mmolL}^{-1}$ ferric (III) chloride solution and with $50 \mathrm{~mL}$ of $0.3 \mathrm{mmolL}^{-1}$ acetate buffer $(\mathrm{pH}=3.6)$. Analysis of FRAP considering cuvettes was performed by adding $0.15 \mathrm{~mL}$ appropriate sample to $0.9 \mathrm{~mL}$ of FRAP reagent.

At the same time, $50 \mu \mathrm{l}$ of respective sample were introduced to well for analysis in microplates. Microplate was put into the spectrophotometer and subsequently $300 \mu \mathrm{l}$ of FRAP reagent was added by injector.

The solution in case of cuvettes and microplates were left for $5 \mathrm{~min}$ at ambient temperature follow by absorbance measurement at $\lambda=593 \mathrm{~nm}$. Trolox was used as a standard for preparation of calibration curve within the range of 0.25 to $250 \mu \mathrm{molL}^{-1}$. Results obtained for the juices were expressed as mmol Trolox Equivalents (TE) per L of juice. All analyses were performed in triplicate. 


\subsection{Oxygen Radical Absorbance Capacity (ORAC)}

The ORAC assay was performed on the basis of the method proposed by Ou et al. [25]. The method was conducted at $37^{\circ} \mathrm{C}$ with usage of fluorescein (working solution $42 \mathrm{nmolL}^{-1}$ ) as the substrate and AAPH $\left(153 \mathrm{mmolL}^{-1}\right.$ ) as an oxygen radical generator prepared in $0.075 \mathrm{molL}^{-1}$ phosphate buffer with $\mathrm{pH}=7.4\left(\mathrm{~K}_{2} \mathrm{HPO}_{4}, \mathrm{NaH}_{2} \mathrm{PO}_{4}\right)$. Aliquots of $2.25 \mathrm{~mL}$ of fluorescein working solution were added into the cuvettes placed in the water bath until the set temperature of $37^{\circ} \mathrm{C}$ was reached. Next, $0.375 \mathrm{~mL}$ of appropriately diluted sample was added followed by measurement of fluorescence at 'time zero'. Subsequently, $0.375 \mathrm{~mL}$ of AAPH solution was added and the reaction was performed during $60 \mathrm{~min}$ with measure of fluorescence every $5 \mathrm{~min}$.

The same samples were analysed in microplates. To each well, $200 \mu$ of fluorescein working solution and $50 \mu 1$ of respective sample were added. Microplate was located into the spectrofluorimeter until the mentioned above temperature was achieved. Subsequently, after the measurement of fluorescence at 'time zero', $50 \mu l$ of AAPH solution was automatically added by injector. Microplate was shaken and the measurement of fluorescence was recorded every 5 min during $120 \mathrm{~min}$.

For both variants, the fluorescence was measured at excitation wavelength $\lambda=493 \mathrm{~nm}$ and emission wavelength $\lambda=515 \mathrm{~nm}$. Trolox within the range from $5 \mu \mathrm{mol} / \mathrm{L}$ to $100 \mu \mathrm{mol} / \mathrm{L}$ was used as a reference standard. Results were presented as mmol Trolox Equivalents (TE) per $\mathrm{L}$ of juice.

\section{Results and discussion}

Presented methods have been chosen on the basis of popularity and importance in the domain of berry research. Quantification of total polyphenols, flavonoids, proanthocyanidins and measurement of antioxidant capacity by application of TEAC by ABTS and TEAC by DPPH as well as FRAP and ORAC methods are broadly applied due to their simplicity and reproducibility. Moreover, these photometric methods are quick and easy to perform. These methods have been widely applied for different berry foodstuffs like fresh fruits, juices [26], jams [27], juice concentrates [28] and wine [12,26-29] creating a huge collection of results. These databases serve to compare newly gained results with existing ones and to assess the quality of berry products.

\subsection{Total phenolic compounds (TPC)}

Chemistry of reaction occurring between polyphenols and Folin-Ciocalteu reagent has been precisely described by Huang et al. [13] and the attention has been drawn to the transfer of electrons from polyphenolic compounds (or other reducing species) to molybdenum during the reaction. Due to this fact the method measures actually the reducing capacity of samples but it is commonly known as total polyphenols. Moreover, from a methodological point of view Prior et al. [14] highlighted different aspects of performance including different concentration of reagents and incubation time that can lead to an overestimation of the obtained results. Hence, the aim of the experiment was to provide insight into the determination of total polyphenols by cuvette and microplate methods leading to the comparison of the content of total polyphenols of different commercial berry juices.

The first part of the experiment aimed to compare calibrations curves prepared on the basis of gallic acid as reference compound for cuvettes and microplates application. Linear regression of both calibrations and correlation coefficients are shown in Table 1. The sensitivity of the method with cuvettes application was higher than for microplates. In our case, the limit of quantification (LOQ) being at the level of $0.015 \mathrm{mg} \mathrm{GAE} / \mathrm{mL}$ was the same for both methods. The tested concentration range of standard was in the linear range of the methods.

The second part of the experiment regarded the determination of polyphenolic compounds in different berry juices. The content of total polyphenolic compounds in juices was within the range from 0.48 to $1.75 \mathrm{gL}^{-1} \mathrm{GAE}$ (Table 3). Acquired data were comparable in case of both methods used for each juice. Obtained results for blueberry, cranberry and açai juices were inline with those obtained by Seeram et al. [30] and Piljac-Žegarac et al. [31]. The highest content of TPC determined by both methods was noted for blueberry juices. Açai was also relatively rich in those compounds compared to cranberry and pomegranate juice. From the practical point of view, over 30 times lower quantities of 
Table 1

Equations of calibration curves for selected methods

\begin{tabular}{llrrr}
\hline Method & & \multicolumn{2}{c}{$y=a x+b$} & \\
\cline { 3 - 4 } & & \multicolumn{1}{c}{$A$} & \multicolumn{1}{c}{$B$} & $\mathrm{R}^{2}$ \\
\hline TPC & Cuvettes & 4.0471 & -0.0305 & 0.999 \\
& microplates & 3.1761 & -0.0125 & 0.999 \\
TF & Cuvettes & 5.4820 & 0.0784 & 0.998 \\
& microplates & 5.0979 & 0.0081 & 0.999 \\
TP & Cuvettes & 1.1307 & 0.0239 & 0.991 \\
& microplates & 1.1209 & 0.0285 & 0.988 \\
TEAC ABTS & Cuvettes & 0.0432 & 0.5755 & 0.999 \\
& microplates & 0.1058 & -0.0163 & 0.997 \\
TEAC DPPH & Cuvettes & 0.0910 & 7.3030 & 0.992 \\
& microplates & 0.0591 & 0.1990 & 0.991 \\
FRAP & Cuvettes & 0.0051 & 0.0701 & 0.995 \\
& microplates & 0.0060 & -0.0143 & 0.999 \\
ORAC & Cuvettes & 0.1971 & 3.0785 & 0.987 \\
& microplates & 0.3738 & 10.106 & 0.996 \\
\hline
\end{tabular}

Table 2

Reagent and time ratios calculated for cuvette and microplate application (cuvettes/microplates)

\begin{tabular}{lcc}
\hline Method & Quantity of reagents & Time of preparation \\
\hline TPC & 33 & 4 \\
TF & 10 & 6 \\
TP & 10 & 5 \\
TEAC ABTS & 5 & 10 \\
TEAC DPPH & 33 & 6 \\
FRAP & 3 & 12 \\
ORAC & 10 & 7 \\
\hline
\end{tabular}

Table 3

Analysis of selected berry juices applying cuvette and microplate methods (mean $\pm \mathrm{SD}, n=3$ )

\begin{tabular}{|c|c|c|c|c|c|c|}
\hline \multicolumn{7}{|c|}{ Juice } \\
\hline Method & & Blueberry & Cranberry & Goji & Açai & Pomegranate \\
\hline \multirow[t]{2}{*}{ TPC [g/L GAE] } & Cuvettes & $1.752 \pm 0.003$ & $1.275 \pm 0.007$ & $1.058 \pm 0.007$ & $1.273 \pm 0.010$ & $0.511 \pm 0.003$ \\
\hline & Microplates & $1.669 \pm 0.050$ & $1.232 \pm 0.031$ & $1.026 \pm 0.004$ & $1.198 \pm 0.062$ & $0.477 \pm 0.008$ \\
\hline \multirow[t]{2}{*}{$\mathrm{TF}[g / \mathrm{L}]$} & Cuvettes & $0.157 \pm 0.004$ & $0.096 \pm 0.004$ & $0.058 \pm 0.002$ & $0.029 \pm 0.000$ & $0.011 \pm 0.001$ \\
\hline & Microplates & $0.150 \pm 0.007$ & $0.097 \pm 0.005$ & $0.062 \pm 0.006$ & $0.032 \pm 0.001$ & $0.025 \pm 0.001$ \\
\hline \multirow[t]{2}{*}{$\mathrm{TP}[\mathrm{g} / \mathrm{L} \mathrm{CAT}]$} & Cuvettes & $2.785 \pm 0.021$ & $1.466 \pm 0.003$ & $0.310 \pm 0.002$ & $0.525 \pm 0.004$ & $0.225 \pm 0.002$ \\
\hline & Microplates & $2.342 \pm 0.091$ & $1.134 \pm 0.038$ & $0.255 \pm 0.025$ & $0.401 \pm 0.071$ & $0.163 \pm 0.005$ \\
\hline
\end{tabular}

reagents are necessary for the microplate application (Table 2). Also, time-consuming performance calculated per 96 samples could be reduces 4 times in case of microplates.

\subsection{Total flavonoids $(T F)$}

According to Chang et al. [19], aluminum chloride forms stable complexes with some major groups of flavonoids (mainly with C4-keto group and C5 or C5 hydroxyl group of flavones and flavonols) which give an absorbance maximum at $\lambda=415 \mathrm{~nm}$. In the literature two applications of this reaction are described. One with $\mathrm{NaNO}_{2}$ [32] and 
another more sensitive working with $\mathrm{CH}_{3} \mathrm{COOK}$ [33]. The authors decided to work with $\mathrm{CH}_{3} \mathrm{COOK}$ due to better sensitivity.

Firstly, two calibrations for cuvettes and microplates were prepared. Linear regression and correlation coefficients are presented in Table 1. Similarly to calibration prepared for TPC, slightly higher sensitivity was noted in case of cuvette method when compared to microplate one. Over the tested concentration range, both methods showed linear correlation. In our case, the LOQ for both methods was $0.015 \mathrm{mgmL}^{-1} \mathrm{Q}$.

Secondly, the content of total flavonoids in commercial berry juices was determined spectrophotometrically by these two methods. Gained results are presented in Table 3. Similarly to TPC, the highest content of TF was noted for blueberry juice determined by both methods (approx. $0.15 \mathrm{gL}^{-1} \mathrm{Q}$ ). Relatively high content of flavonoids was noted for cranberry and goji juices when compared to blueberry one. The lowest content of flavonoids among the analysed juices was noted for pomegranate and açai. From practical point of view, the performance of this method could be shorted 6 times with a reduction of reagents down to 10-fold (Table 2).

\subsection{Total proanthocyanidins $(T P)$}

The chemistry of the methods relies on a condensation reaction between vanillin and proanthocyanidins under acidic condition. Protonated vanillin reacts with 6 or 8 position on the A ring of any flavan-3-ol subunit. The intermediate products of this reaction gives a colour from light pink to deep red [20]. This colour change might be followed by absorbance measurement at $\lambda=500 \mathrm{~nm}$. Linear regression and correlation coefficients of both methods for $( \pm)$-catechin are shown in Table 1 . The sensitivity and the limit of quantification $\left(0.015 \mathrm{mgmL}^{-1} \mathrm{CAT}\right)$ were at similar level.

Among analysed berry products, the highest content of total proanthocyanidins was noted for blueberry juice followed by cranberry juice. The lowest content of proanthocyanidins was observed for pomegranate juice (Table 3 ). In the literature there is scarce information about the content of total proanthocyanidins in commercial berry juices and most results concern fresh fruits [33-34].

\subsection{Determination of antioxidant capacity}

\subsubsection{TEAC by ABTS}

Trolox Equivalent Antioxidant Capacity with $\mathrm{ABTS}^{\bullet+}$ as a free radical and $\mathrm{K}_{2} \mathrm{~S}_{2} \mathrm{O}_{8}$ as oxidizing agent gained popularity due to broad range of application used for different foodstuffs. The mechanism of reaction has been described by hydrogen atom transfer and at the same time single electron transfer [13, 14]. Many variations for this method regarding the reaction time as well as wavelength applied have been shown $[35,36]$.

The dose-response curve gained by the analysis of different concentrations of Trolox was in the linear range for both cuvette and microplate methods (Table 1). Limit of detection for cuvettes was at the level of $25 \mu \mathrm{molL}{ }^{-1} \mathrm{Trolox}$, whereas for microplates it was at $7 \mu \mathrm{molL}^{-1}$ Trolox. The sensitivity of TEAC by ABTS was higher for microplate method then for cuvette one (Table 1).

Subsequently, the ability to scavenge ABTS $^{\bullet+}$ radical cation was investigated in the selected berry juices and it was within the range from 4.11 to $16.62 \mathrm{mmol} / \mathrm{L}$ Trolox (Table 4). The highest antioxidant capacity measured by TEAC ABTS method was noted for blueberry juices performed by both methods. The results were comparable to those obtained by [30] with application of microplates. Next, the antioxidant capacity of tested juices was in the order: cranberry>açai $>$ goji $>$ pomegranate. The same tendency was observed in case of cuvette method. Gained results for microplate were slightly lower than those obtained by application of cuvettes. This could be due to the different reagent quantities used. Taking into account that the $\mathrm{ABTS}^{\bullet+}$ solution was diluted until absorbance at $\lambda=734 \mathrm{~nm}$ was $0.7( \pm 0.05)$, different volumes of $\mathrm{ABTS}^{\bullet+}$ working solution were used for analysis in case of cuvettes (sample: ABTS $^{\bullet+}, 20 \mu \mathrm{l}: 1480 \mu \mathrm{l}$ ) and for microplates (sample: ABTS ${ }^{\bullet+}, 10 \mu \mathrm{l}: 290 \mu \mathrm{l}$ ). From the practical point of view, use of microplates leads to the reduction of preparation time down to 10 times and 5 times less reagents are required (Table 2). 


\subsubsection{TEAC by $D P P H$}

DPPH is a stable organic radical that has been widely used for determination of antioxidant capacity. The chemistry of this method as well as advantages and disadvantages are described in the literature [13,14]. For this method also Trolox has been used as reference compound. The calibrations for cuvette and microplate methods were prepared with limit of determination at $50 \mu \mathrm{molL}^{-1}$ Trolox. The linear regression is presented in Table 1 . Highest sensitivity was noted in case of cuvettes.

Antioxidant capacity of investigated juices measured by DPPH method is presented in Table 4 . The highest ability to scavenge $\mathrm{DPPH}^{*}$ had blueberry and açai juices. Relatively lower antioxidant capacity was noted for cranberry, goji and pomegranate juices when compare to previous ones. Determination of antioxidant capacity by microplates may reduce reagents 33 times and decrease the preparation time 6 fold (Table 2).

\subsubsection{FRAP}

Ferric Reducing Antioxidant Power (FRAP) measures the reduction of 2,4,6-tripyridyl-s-triazine (TPTZ) to a blue coloured product. The reaction is performed in acidic condition and is commonly used for biological matrices [14]. In our experiment the FRAP method has been used for preparation of two calibrations curves with Trolox as a reference compounds. Linear regressions for cuvette and microplate method are presented in Table 1. The sensitivity was at the same level for both methods. The limit of determination for both methods was at $0.12 \mu$ molL ${ }^{-1}$ Trolox.

Obtained results of FRAP assay performed in commercial berry juices are presented in Table 4. Among analysed juices, the highest values were noted for blueberry juice in case of cuvettes $\left(14.41 \mathrm{mmolL}^{-1}\right.$ Trolox) and microplates $\left(12.18 \mathrm{mmolL}^{-1}\right.$ Trolox). Relatively lower values were indicated for analysed juices in order: açai $>$ cranberry $>$ goji $>$ pomegranate. Application of microplate method can diminished the usage of reagents 3 times and could decrease the analysis time almost 12 times (Table 2).

\subsubsection{ORAC}

Oxygen Radical Antioxidant Capacity is one of the most popular method used for determination of antioxidant capacity of biological samples. Chemistry of reaction that occurs between fluorescein and antioxidants presented in an analysed sample has been broadly described in the literature [13, 14, 37].

The dose-response curve based on Trolox as a reference compound was prepared for cuvette and microplate method. The calibrations were prepared concerning the concentration of Trolox against area under the fluorescence decay curve (AUC) for fluorescein. Linear regression and correlation coefficient are presented in Table 1. Higher sensitivity was noted in case of microplate method when compare to the cuvette one (Table 2).

In the second part of the experiment, berry juices have been subjected to analysis of antioxidant capacity and gained results are presented in Table 4 . The antioxidant capacity measured by ORAC method was from 6.15 to $34.25 \mathrm{mmolL}^{-1}$ Trolox. Among tested juices, the highest ability to scavenge ROO was noted for blueberry juice. Obtained results regarded to blueberry, açai, cranberry juice were comparable to those gained by [30]. Approx. 50\% lower antioxidant capacity was observed for cranberry and goji juices when compare to blueberry. Moreover, the lowest ability for scavenging ROO was noted for pomegranate juice. Preparation of ORAC in microplates might

Table 4

Antioxidant capacity of selected berry juices applying cuvette and microplate methods

\begin{tabular}{llrrrrr}
\hline \multirow{2}{*}{ Method } & Juice & Blueberry & Cranberry & Goji & Açai & Pomegranate \\
\hline TEAC ABTS & Cuvettes & $16.620 \pm 0.240$ & $10.925 \pm 0.195$ & $8.347 \pm 0.469$ & $9.282 \pm 0.117$ & $4.692 \pm 0.195$ \\
& microplates & $15.098 \pm 0.223$ & $11.502 \pm 0.427$ & $7.727 \pm 0.176$ & $9.290 \pm 0.253$ & $4.112 \pm 0.150$ \\
TEAC DPPH & Cuvettes & $17.390 \pm 0.043$ & $13.732 \pm 0.059$ & $10.624 \pm 0.049$ & $17.352 \pm 0.097$ & $5.412 \pm 0.037$ \\
& microplates & $16.867 \pm 0.059$ & $10.573 \pm 0.239$ & $8.567 \pm 0.043$ & $17.512 \pm 0.351$ & $4.264 \pm 0.043$ \\
FRAP & Cuvettes & $14.408 \pm 0.424$ & $8.096 \pm 0.006$ & $5.117 \pm 0.374$ & $10.916 \pm 0.104$ & $3.146 \pm 0.277$ \\
& microplates & $12.181 \pm 0.393$ & $6.774 \pm 0.133$ & $4.386 \pm 0.083$ & $8.561 \pm 0.080$ & $2.888 \pm 0.023$ \\
ORAC & Cuvettes & $34.250 \pm 1.076$ & $18.646 \pm 0.387$ & $14.652 \pm 0.054$ & $15.096 \pm 0.341$ & $7.206 \pm 0.187$ \\
& microplates & $34.070 \pm 0.481$ & $17.334 \pm 0.052$ & $15.743 \pm 0.254$ & $14.156 \pm 0.206$ & $6.149 \pm 0.020$ \\
\hline
\end{tabular}

Values are given as mmol Trolox/L and are mean $\pm \mathrm{SD}, n=3$. 
Table 5

Correlation coefficients of the methods for cuvette and microplate application

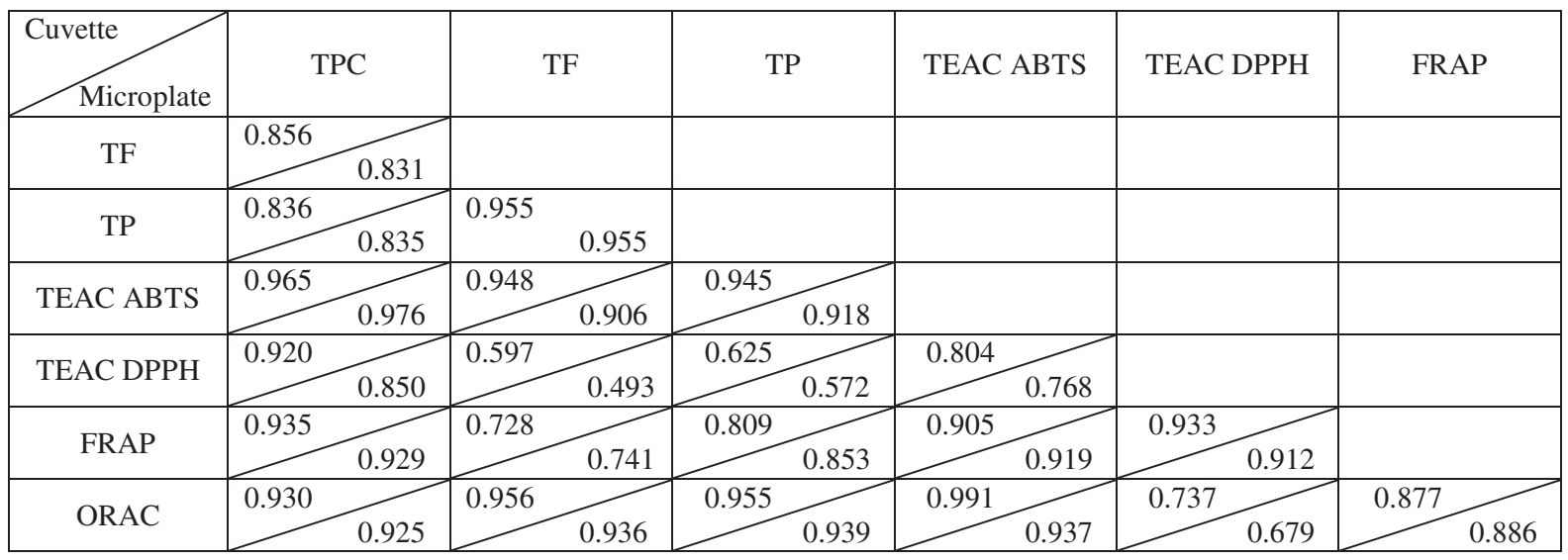

reduce the usage of solvents 10 times and shorter the time of analysis 7-fold (Table 2). Similar comparison has been made by Huang et al. [38] where authors improved efficiency of the method 10-fold.

Different methods to analyse antioxidants give different results (Table 4), due to different reaction mechanisms of the antioxidants present in berry fruits. Thus, there is no universal method available for determination of antioxidant capacity of fruits samples [39-41]. Therefore, it is pertinent to apply not only one method but a spectrum of methods to characterise a sample as best as possible. However, in the present study comparing fruit juices, the methods are relatively good correlated for (Table 5). The lower correlation for TEAC DPPH with all the other methods is remarkable, and relies on a different reactivity of DPPH with the antioxidants present in the juices. Also, it is worth to mention critical points which could strongly influence determination of antioxidant capacity of berry samples. Scalzo et al. [42] highlighted that values of antioxidant capacity may differ not only due to the variety of berry fruits, but also due to extraction procedure for sample preparation and choice of appropriate method.

Adaptation of the selected methods to microplate measurement has been performed showing similarity and some differences in obtained results. Indeed, automation of the presented methods by using equipment with injectors causes reduction of reagent quantities and preparation time (Table 2). Both aspects influence the efficiency of analyses mainly in case of big numbers of samples. In our study, the comparison had been made in case of preparation of 40 samples, two repetitions including calibration curves and reference samples. Moreover, comparison of the costs concerning usage of microplate and cuvettes indicate that expenses for microplates could be reduced 10 times in case of performing experiment for 96 samples. Looking at overall analysis costs, price for a UV/Vis photometer, fluorimeter and a microplate reader have to be considered. In the present study a microplate reader which was able to measure absorption and fluorescence was used. In general, acquisition costs of a microplate reader is about five times higher than that of a photometer, but this depends highly on equipment of the individual analytical devices.

\section{Conclusions}

Popular and important photometric methods for the assessment of berry quality have been selected and adapted from cuvette to microplate application. With five commercial berry juices methods have been shown to be appropriate for a fast and cheap quality assessment.

These cheap and quick microplate methods support plant breeders for the selection of promising varieties, rich in bioactive compounds, for commercial fruit and vegetable production. Based on the selected high quality plants, the producers and indirectly the consumers will profit from a higher fruit and vegetable quality.

Five commercial berry juices were employed for determination of bioactive compounds and antioxidant capacity by methods that could be easily used to screen and evaluate the quality of the commercially available products. The application of more sophisticated methods required advanced equipment and a prescreening with simple methods 
is appropriate and highly recommended. Presented comparison between traditional photometric methods and their modern microplate versions could be useful for fast prescreening bioactive compounds content and antioxidant capacity performed in a small laboratories giving information for breeders and also consumers. Therefore, different juices were used in order to present applicability of microplate usage in comparison to cuvette ones.

\section{Acknowledgments}

Authors are grateful to CRUS (Rectors' Conference of the Swiss Universities) for financial support of Anna Horszwald (SCIEX fellowship No. PL0908002, Scientific Exchange Programme NMS-CH).

\section{References}

[1] N.P. Seeram, Berry fruits: compositional elements, biochemical activities, and the impact of their intake on human health, performance, and disease, J Agric Food Chem 56(3) (2008), 627-629.

[2] G.D. Stoner, L.S. Wang and B.C. Casto, Laboratory and clinical studies of cancer chemoprevention by antioxidants in berries, Carcinogenesis 29(9) (2008), 1665-1674.

[3] Food and Agriculture Organization of the United Nations. Faostat; 2010 [cited 2010 Dec 12]. Available from www.faostat.fao.org

[4] N.P. Seeram, Berry fruits for cancer prevention: Current status and future prospects, J Agric Food Chem 56(3) (2008), 630-635.

[5] H.-D. Belitz, W. Grosch and P. Schieberle, Food Chemistry, Third edition ed, H. Springer Berlin (ed.), Springer, New York.

[6] N.P. Seeram, L.S. Adams, Y.J. Zhang, R. Lee, D. Sand and H.S. Scheuller, Blackberry: black raspberry, blueberry, cranberry, red raspberry, and strawberry extracts inhibit growth and stimulate apoptosis of human cancer cells in vitro, J Agric Food Chem 54(25) (2006), 9329-9339.

[7] W. Andlauer and P. Furst, Antioxidative power of phytochemicals with special reference to cereals, Cereal Food World 43(5) (1998), $356-360$.

[8] W. Andlauer, P. Stehle and P. Furst, Chemoprevention-a novel approach in dietetics, Curr Opin Clin Nutr Metab Care 1(6) (1998), $539-547$.

[9] S. Zafra-Stone, T. Yasmin, M. Bagchi, A. Chatterjee, J.A. Vinson and D. Bagchi, Berry anthocyanins as novel antioxidants in human health and disease prevention, Mol Nutr Food Res 51(6) (2007), 675-683.

[10] M. Battino, J. Beekwilder, B. Denoyes-Rothan, M. Laimer, G.J. McDougall and B. Mezzetti, Bioactive compounds in berries relevant to human health, Nutr Rev 67(5) (2009), 145-150.

[11] E. Niki, Assessment of antioxidant capacity of natural products, Curr Pharm Biotechnol 11(8) (2010), 801-809.

[12] A. Hartmann, C.D. Patz, W. Andlauer, H. Dietrich and M. Ludwig, Influence of processing on quality parameters of strawberries, J Agric Food Chem 56(20) (2008), 9484-9489.

[13] D.J. Huang, B.X. Ou and R.L. Prior, The chemistry behind antioxidant capacity assays, J Agric Food Chem 53(6) (2005), $1841-1856$.

[14] R.L. Prior, X.L. Wu and K. Schaich, Standardized methods for the determination of antioxidant capacity and phenolics in foods and dietary supplements, J Agric Food Chem 53(10) (2005), 4290-4302.

[15] L.M. Magalhaes, M. Santos, M.A. Segundo, S. Reis and J.L. Lima, Flow injection based methods for fast screening of antioxidant capacity, Talanta 77(5) (2009), 1559-1566.

[16] L.M. Magalhaes, M.A. Segundo, S. Reis, J.L. Lima, I.V. Toth and A.O. Rangel, Automatic flow system for sequential determination of ABTS $^{*+}$ scavenging capacity and Folin-Ciocalteu index: a comparative study in food products, Anal Chim Acta 592(2) (2007), $193-201$.

[17] W. Andlauer and J. Heritier, Rapid electrochemical screening of antioxidant capacity (RESAC) of selected tea samples, Food Chem 125(4) (2011), 1517-1520.

[18] T. Siriwoharn, R.E. Wrolstad, C.E. Finn and C.B. Pereira, Influence of cultivar: maturity, and sampling on blackberry (Rubus LHybrids) anthocyanins, polyphenolics, and antioxidant properties, J Agric Food Chem 52(26) (2004), 8021-8030.

[19] C.C. Chang, M.H. Yang, H.M. Wen and J.C. Chern, Estimation of total flavonoid content in propolis by two complementary colorimetric methods, J Food Drug Anal 10(3) (2002), 178-182.

[20] B.S. Sun, J.M. Ricardo-da-Silva and I. Spranger, Critical factors of vanillin assay for catechins and proanthocyanidins, J Agric Food Chem 46(10) (1998), 4267-4274.

[21] R. Re, N. Pellegrini, A. Proteggente, A. Pannala, M. Yang and C. Rice-Evans, Antioxidant activity applying an improved ABTS radical cation decolorization assay, Free Radic Bio Med 26(9-10) (1999), 1231-1237.

[22] E.M. Silva, J.N.S. Souza, H. Rogez, J.F. Reesb and Y. Larondellea, Antioxidant activities and polyphenolic contents of fifteen selected plant species from the Amazonian region, Food Chem 101(3) (2007), 1012-1018.

[23] W. Brand-Williams, M.E. Cuvelier and C. Berset, Use of a Free-Radical method to evaluate antioxidant activity, LWT - Food Sci Technol 28(1) (1995), 25-30. 
[24] I.F.F. Benzie and J.J. Strain, The ferric reducing ability of plasma (FRAP) as a measure of "antioxidant power": The FRAP assay, Anal Biochem 239(1) (1996), 70-76.

[25] B.X. Ou, M. Hampsch-Woodill and R.L. Prior, Development and validation of an improved oxygen radical absorbance capacity assay using fluorescein as the fluorescent probe, J Agric Food Chem 49(10) (2001), 4619-4626.

[26] E. Balogh, A. Hegedus and E. Stefanovits-Banyai, Application of and correlation among antioxidant and antiradical assays for characterizing antioxidant capacity of berries, Sci Hortic 125(3) (2010), 332-336.

[27] T. Wicklund, H.J. Rosenfeld, B.K. Martinsen, M.W. Sundfor, P. Lea and T. Bruun, Antioxidant capacity and colour of strawberry jam as influenced by cultivar and storage conditions, LWT - Food Sci Technol 38(4) (2005), 387-391.

[28] M.J. Bermudez-Soto and F.A. Tomas-Barberan, Evaluation of commercial red fruit juice concentrates as ingredients for antioxidant functional juices, Eur Food Res Technol 219(2) (2004), 133-141.

[29] M.S. Su and P.J. Chien, Antioxidant activity: anthocyanins, and phenolics of rabbiteye blueberry (Vaccinium ashei) fluid products as affected by fermentation, Food Chem 104(1) (2007), 182-187.

[30] N.P. Seeram, M. Aviram, Y. Zhang, S.M. Henning, L. Feng and M. Dreher, Comparison of antioxidant potency of commonly consumed polyphenol-rich beverages in the united states, J Agric Food Chem 56(4) (2008), 1415-1422.

[31] J. Piljac-Žegarac, L. Valek, S. Martinez and A. Belščak, Fluctuations in the phenolic content and antioxidant capacity of dark fruit juices in refrigerated storage, Food Chem 113(2) (2009), 394-400.

[32] Z. Jia, M. Tang and J. Wu, The determination of flavonoid contents in mulberry and their scavenging effects on superoxide radicals, Food Chem 64(4) (1999), 555-559.

[33] J.Y. Lin and C.Y. Tang, Determination of total phenolic and flavonoid contents in selected fruits and vegetables: as well as their stimulatory effects on mouse splenocyte proliferation, Food Chem 101(1) (2007), 140-147.

[34] Y.F. Li, C.J. Guo, J.J. Yang, J.Y. Wei, J. Xu and S. Cheng, Evaluation of antioxidant properties of pomegranate peel extract in comparison with pomegranate pulp extract, Food Chem 96(2) (2006), 254-260.

[35] M.B. Arnao, Some methodological problems in the determination of antioxidant activity using chromogen radicals: a practical case, Trends Food Sci Technol 11(11) (2000), 419-421.

[36] S. Bompadre, L. Leone, A. Politi and M. Battino, Improved FIA-ABTS method for antioxidant capacity determination in different biological samples, Free Radical Res 38(8) (2004), 831-838.

[37] G.H. Cao, H.M. Alessio and R.G. Cutler, Oxygen-Radical Absorbency Capacity assay for antioxidants, Free Radical Bio Med 14(3) (1993), 303-311.

[38] D.J. Huang, B.X. Ou, M. Hampsch-Woodill, J.A. Flanagan and R.L. Prior, High-throughput assay of oxygen radical absorbance capacity (ORAC) using a multichannel liquid handling system coupled with a microplate flourescence reader in 96-well format, $J$ Agric Food Chem 50(16) (2002), 4437-4444.

[39] E. Niki, Assessment of antioxidant capacity in vitro and in vivo, Free Radic Biol Med 49(4) (2010), 503-515.

[40] Y. Omata, Y. Yoshida and E. Niki, Assessment of the antioxidant capacity of natural fruit extracts by inhibition of probe decay and plasma lipid peroxidation, Biosci Biotechnol Biochem 74(3) (2010), 531-535.

[41] Y. Omata, Y. Saito, Y. Yoshida and E. Niki, Simple assessment of radical scavenging capacity of beverages, J Agric Food Chem 56(9) (2008), 3386-3390

[42] J. Scalzo, B. Mezzetti and M. Battino, Total antioxidant capacity evaluation: critical steps for assaying berry antioxidant features, Biofactors 23(4) (2005), 221-227. 